\title{
Riesgo y Razón: seguridad, ley y medioambiente. 2006. Sunstein, C. Buenos Aires, Editorial Katz.
}

Revisado por

Maximiliano E. Korstanje

International Research Commitee on Disasters, Texas USA

http://dx.doi.org/10.5209/rev_NOMA.2014.v42.n2.48776

A días del accidente ferroviario en estación Once (en la ciudad de Buenos Aires, Argentina) que le quitara la vida a 51 personas y dejara centenares de heridos, el libro de Cass Sunstein se presenta como un desarrollo profundo y sistemático por responder a dos preguntas bien distintas; ¿por qué cada vez tenemos más miedo?, ¿porque nuestro miedo no lleva a políticas públicas eficientes?. Para abordar estas cuestiones, Sunstein trae como ejemplo el pánico generado en Washington DC respecto al francotirador que asesinara (al azar) a diez ciudadanos americanos. Si bien el temor, en ese contexto, ha sido muy intenso, hasta el punto de modificar las conductas particulares y hábitos en la vida cotidiana, la paranoia puede generar mayores complicaciones. Por lo general, estamos preocupados por el SARS, los desastres naturales o el terrorismo sin darnos cuenta que subestimamos prácticas muy concretas como ser la alimentación o el consumo de tabaco que llevan anualmente a miles de personas a la muerte. Este será el tema central de toda la obra, discutida a través de diferentes ejemplos y análisis de casos ilustrativos.

Según lo expuesto, Sunstein advierte que existen dos mecanismos por los cuales se maximizan riesgos menores y se desatienden otros de mayor impacto: a) la heurística de la disponibilidad y b) el descuido de la probabilidad. El primer mecanismo se refiere al uso mental de episodios similares disponibles con facilidad pero que mantienen una baja probabilidad de concreción. Cuando ello sucede, la opinión pública sobrevalora el riesgo generando un estado de alarma extendida. Por el contrario, el descuido de la probabilidad se activa cuando la gente se muestra altamente sensible a los efectos del riesgo, descuidando las probabilidades. Sunstein aclara que "cuando las emociones" afloran, se descuida la probabilidad. En pánico generalizado parece ser resultado de una combinación entre ambos mecanismos, que fagocitados por los medios de comunicación, crean estados emocionales específicos que desestabilizan el sistema político. Si la ciudadanía pide mayor seguridad, el Estado empleará los medios a su disposición para dar mayor seguridad, pero esa medida no solo será ineficiente, sino que puede crear nuevos peligros. Estas políticas (populistas) no llevan a la solución del problema.

El experimento de Dorner, precisamente, demuestra (por medio de la proyección computarizada) como incluso las decisiones de los expertos generan calamidades. El proceso decisorio no está sujeto a una visión holística y abarcadora del fenómeno, y lo que se arregla en un sentido, se destruye en otro. En el año 2000, la ciudad de Hatfield (en el Reino Unido) fue protagonista de un accidente ferroviario que puso en vilo a la opinión pública británica hasta el punto que más de la tercera parte de los usuarios habituales empezaron a 
usar automóviles y buses para desplazarse. Este sentimiento generalizado de miedo no solo no resolvió satisfactoriamente el problema, sino que por la cantidad de unidades circulando aumentó significativamente la cantidad de muertos en las rutas de ese país. La lectura del caso, nos demuestra, que las rutas inglesas terminaron siendo potencialmente más inseguras que los ferrocarriles. Este aspecto es una de las preocupaciones más evidentes en el desarrollo de Sunstein. ¿Cómo hacer que el Gobierno atienda a las verdaderas causas de los riesgos que potencialmente son disfuncionales para la sociedad?.

En los capítulos introductorios, el autor examina las paradojas de la complejidad y como en ocasiones el Estado falla en controlar los riesgos, precisamente no por lo que deja de hacer, sino por las políticas que implementa. El problema se da cuando la mitigación de un riesgo, aumenta las probabilidades de sufrir otro peor. Sunstein afirma que sólo el modelo de costobeneficio puede ayudar al estado a tomar una decisión racional y eficiente frente al peligro. El estado debería implementar un plan de acción cuando la regulación justifica sus costes. Es evidente el escepticismo del libro respecto de la posibilidad que el ciudadano tiene para tomar una decisión correcta. Los riesgos son más un producto de las malas decisiones que de causas externas al sujeto. En este punto de análisis Sunstein escribe:

"La gente recurre a atajos mentales que a menudo funcionan bien pero que también pueden producir grandes errores. La gente adopta una suerte de toxicología intuitiva que lleva a temores insoportables. Sus emociones la pueden descaminar. En demasiados casos no ve la necesidad de transacciones. Es susceptible a influjos sociales que llevan a cascadas de temor $y$ de descuido. Los grupos de interés bien organizados, en la industria y la comunidad del interés público, se hallan más que dispuestos a explotar las limitaciones cognitivas y los influjos sociales en su beneficio. En estas circunstancias, la ventaja principal del análisis de costo-beneficio es que puede poner sobre la mesa los efectos de diferentes enfoques, y de esa manera ayudar a acicatear la acción gubernamental". (p. 30)

El texto se encuentra estructurado en diez capítulos donde se defiende el modelo costo-beneficio aplicado a la reducción de riesgos ambientales. Si por algún momento, pensamos en cambiar el tipo de combustible para emitir menos dióxido de carbono a la atmosfera, debemos tener en cuenta todas las variables pero por sobre todo, los costos que un cambio de política de este tipo implica. La tesis del profesor Sunstein, a diferencia de Giddens, se ubica en un racionalismo moderno en donde se cuestiona el rol de la cadena de expertos y sus juicios de valor. El mercado no sería un elemento suficiente para corregir por si mismo los desequilibrios ecológicos. El hombre desarrolla su pensamiento en base a ideas equivocadas las cuales deben ser reformuladas, y controladas por un Estado racional. Empero ¿no está ese Estado formado por los mismos hombres que Sunstein menciona?.

Alternativamente, la repuesta versa sobre la siguiente dirección. El Estado y el sistema de leyes son los encargados de cuidar por la seguridad de los 
ciudadanos. La norma en una democracia fuerte, tiene el poder suficiente para poder regular actos que atentan contra la seguridad general. Respecto a las disonancias cognitivas de la opinión pública, tanto el Congreso como los jueces pueden sancionar una ley que trabaje por "excepción"; ello se remite a la idea de no intervenir en riesgos menores los cuales no representan un peligro para la sociedad (aunque sean magnificados por los medios). El conflicto político que puede derivarse de estas excepciones llevará indefectiblemente a una "transacción de riesgos". En consecuencia, las cuestiones que por un lado implican riesgos, por el otro traen beneficios significativos. Anularlas por completo sería (en eso coincide con Giddens) abrir nuevos riesgos no contemplados. Como esa cadena racional es imposible de quebrar, es necesario explorar, estudiar, comprender y comparar cual es el mal menor (riesgo sustituto). El autor admite, se da una injustificada crítica sobre su modelo que puede resumirse en las siguientes líneas:

a) La magnitud de los costos no es tan importante para la vida social. La regulación no contempla la posición de los estratos más pobres.

b) El modelo costo beneficio trivializa el rol de la incertidumbre en la toma de decisiones.

c) Los bienes sociales no deben evaluarse como bienes patrimoniales.

d) El modelo explica pero no reduce la sensación de miedo generada por la "toxicología intuitiva".

e) Pensar la política desde la reducción del riesgo, es entrar en una especie de dictadura de los expertos.

f) El modelo costo beneficio nace del cálculo y se corresponde como una forma ideológica del capitalismo.

A todas estas críticas, Sunstein responde que en muchos sentidos se debe abogar por una legislación tecnocrática de los riesgos simplemente porque de ellos depende no solo la calidad de vida del ciudadano, sino la democracia. Es necesario, vencer el avance del "populismo" y de discursos irracionales que comprometen los recursos de la sociedad a largo plazo. El estado no debe incurrir en demagogia apelando a gastar sus recursos en todo lo que sus ciudadanos demandan. La educación, en este contexto, es la herramienta indicada para generar confianza en la función pública. En lo personal, el trabajo de Sunstein se encuentra orientado a explicar dos aspectos importantes del riesgo, su constitución y percepción. Su solidez académica es incuestionable y refleja años de experiencia en el tema; Riesgo y Razón es una obra equiparable a los trabajos más representativos en la materia. Empero si uno se detiene a examinar críticamente los alcances de la obra, debe reparar en dos limitaciones centrales.

La primera y más importante, es que Sunstein intenta explicar un fenómeno sin abstraerse por completo de la dinámica que el mismo fenómeno impone. El riesgo es un resultado, como adhiere Giddens, de la modernidad y la expansión del capital. En ese contexto, el riesgo es la formula misma del cálculo y no de la ignorancia del pueblo. Siguiendo este razonamiento, el modelo costo beneficio estaría condenado a generar mayores riesgos y no a reducirlos. Básicamente, la segunda crítica radica en la posición utópica respecto a la democracia. El autor asume in facto esse que Estados Unidos es la cuna de la "democracia 
ideal" en donde por deliberativa la función de sus instituciones se encuentran saneadas del peligro que representa el "populismo". Una minuciosa revisión historiográfica nos permitirá comprender que la democracia moderna anglosajona no solo dista bastante de asemejarse a la griega, sino que es propia de una forma de organización anclada en el consumo. La libertad masiva como tal, ha sido una construcción originada en la revolución industrial para que el trabajador pudiera vender su fuerza de trabajo a uno o más de un empleador. De esta forma, la sociedad movilizó fuerzas (hasta ese entonces reprimidas por la caridad católica) para desestructurar los vínculos sociales y con ellos las lealtades de los ciudadanos. Con el advenimiento del Estado nacional, el maquinismo, la separación entre tiempo y espacio y la democracia moderna, el dinero comenzó a funcionar como un mediador de las relaciones, amplificando las potencialidades del riesgo (conectando también espacios descolonizados e inconexos). Las compañías de seguro, creadas para absorber los peligros del ambiente a cambio de una cuota de capital y el interés aplicado a los negocios (que nace con la Reforma) son dos de los elementos que utiliza el capitalismo para reproducirse.

Además, cada sociedad desarrolla formas específicas de vivir la democracia. Por ejemplo, mientras los países anglosajones enfatizan en la funcionalidad de sus instituciones y en su autonomía para resolver problemas, o reducir sus peligros, los latinoamericanos prefieren recurrir a la eficacia de sus gobernantes (instrumentalismo personalista). El primer subtipo se detiene en el proceso, el segundo en la eficiencia o resultado final. Para los anglosajones, su preocupación por la institucionalidad ha llevado a una configuración corporativa de la democracia, con ecazo índice de conflicto social gracias a su apego a la institucionalidad. Por el contrario, en el caso de los hispanoamericanos su concepción ha generado "partidocracia débiles" con un alto sesgo "personalista" acompañadas de una alta conflictividad social e inestabilidad. Eso de ninguna manera autoriza a afirmar que Estados Unidos es más democrático que Argentina o viceversa.

Segundo y desde una perspectiva antropológica, el riesgo debe ser comprendido como una categoría social que ayuda a la reproducción del capital y al sustento del orden económico. Todo riesgo, como el tabú, opera gracias a una prohibición, un mensaje moral distribuido a todos los ciudadanos por medio de la comunicación, la religión y el periodismo. Estas cláusulas recanalizan no solo a la fuerza de trabajo (oferta) sino también las posibilidades de la demanda, produciendo bienes específicos. Comparativamente, el riesgo opera confiriendo a ciertos grupos y bienes únicos (que esos ellos producen) un mayor valor. Dicho valor permite una depreciación de otros bienes los cuales son comercializados al resto de la población, generando un "desequilibrio en las relaciones de poder". Vamos a un ejemplo claro que bien trata Sunstein, "el terrorismo" como forma de riesgo adquiere una característica negativa para los estadounidenses; y particularmente, también una significación específica que difiere de otros países. El discurso del terrorismo prohíbe ciertas prácticas, consumos y bienes a la vez que estimula otros. Aumenta los seguros, pero reduce la circulación de armas entre la población o de elementos que pueden ser considerados "peligrosos". Al reducirse la demanda de esos bienes "prohibidos" su valor se encarece, y a su vez, es controlado bajo el monopolio 
de las elites locales. De esta forma, la población en general se sentirá satisfecha de cubrir sus necesidades de seguridad cotidianas gracias a la contratación de ciertos bienes o seguros, dejando al estado el monopolio de los bienes-tabú. En otras palabras, el riesgo corta la interacción en un sentido y la redirecciona en otro según los intereses políticos. El riesgo disciplina a los agentes sociales, pero lo que es más importante, permite reducir la ambigüedad normativa para que la sociedad pueda seguir produciendo. Se puede afirmar, en resumen, que el problema en Sunstein (similar al desarrollo en Giddens y a muchos pensadores anglosajones) se ubica en la siguiente quimera, no puede hacerse frente a los riesgos por más potencialmente destructivos que ellos sean con la democracia deliberativa o con el cálculo racional porque ambos son las dos caras de una misma moneda; ese parece ser el punto más débil del completo enfoque revisado en la presente pieza. Al margen de lo expuesto, Riesgo y Razón es un trabajo titánico, de una seriedad académica incuestionable y ampliamente recomendable para especialistas en temas de riesgo. 